\title{
8
}

\section{Accommodating Health}

\author{
Bernadette M. Watson
}

\section{Liz Jones}

\section{David G. Hewett}

Health care is fundamental to modern society, but is complex and unsafe (e.g., Vogus, Sutcliffe, \& Weick, 2010), with worldwide acknowledgement that health communication failures are the leading cause of error and adverse events in health (Leonard, Graham, \& Bonacum, 2004; Ong \& Coiera, 2011). Our focus here is to demonstrate how communication accommodation theory (CAT) assists in understanding the underlying causes of ineffective health communication in all contexts, and consider the applied potential for CAT in improving health care education and delivery.

We begin with an overview of early research where CAT was invoked to examine health professional and patient interactions (Watson \& Gallois, 2007), and then review more recent research in which the CAT health focus has expanded to investigate interspecialty communication between doctors and interprofessional communication more generally. We describe how health professionals communicate and negotiate patient care and how CAT explains how, when and why miscommunication can occur both during traditional dyadic encounters, but also within and between multidisciplinary health care teams. 
The chapter closes by revisiting the theoretical basis of CAT in health research and suggesting new directions. We will argue that CAT is well positioned to not only explicate the complexity of health care encounters, but to also improve health care through the design and delivery of interventions that move beyond skills based training.

\section{Health Care as an Intergroup Context}

In this chapter, our fundamental premise is that health care interactions are interpersonal interactions that occur at an intergroup level (see Chapter 7). CAT constructs interpersonal interactions as grounded in the social identity(ies) of the interactants, and predicts that communication is influenced by the "group" memberships that are salient for each participant. In health care, these identities/groups are those of patients and a range of health professionals (Street \& Wiemann, 1988). Status differentials between interactants shape their communication, and in doctor-patient interactions, for example, the doctor has high status.

This intergroup context is pervasive in health, particularly hospitals, due to the very traditional, strongly hierarchical, and highly role-bound system that governs patient care, where individuals identify themselves in their professional roles (e.g., doctors, nurses), each with their own language and cultures (Watson, Gallois, Hewett, \& Iones, 2012; Watson, Hewett, \& Gallois, 2012). A strong identification with these roles and the related status differences influences the communication that occurs between health professionals about patient care, and creates a "silo" mentality impacting on patient care (see Kreindler, Dowd, Dana 
Star, \& Gottschalk, 2012; Peters, Morton, \& Haslam, 2010). Such silos (another metaphor for the intergroup climate) apply to all areas of the hospital system and not just clinicians, but also managers responsible for clinical governance, and human and financial resources, and administrative and technical staff (Coiera, 2011). Until the beginning of the twenty-first century, the intergroup dimension of health communication tended to be ignored by health communication scholars, who explained miscommunication between individuals as due to communication skills deficits that could, in turn, be rectified with training (Wright, Sparks, \& O'Hair, 2008). Such a perspective on communication competence comes from traditional intercultural communication research (Emry \& Wiseman, 1987), and focuses on training individuals in specific communication skills to assist with effective communication, including management of difficult situations. The naivety of this approach was noted by Cargile and Giles (1996), who proposed that intergroup relations are an important component of an interaction. In health, focusing on communication competence does not recognize that individuals possess social and professional identities that are often highly salient during their health care interactions. Communication skills training, by itself, does not address how individuals understand and negotiate their roles with other health professions and with patients.

With recognition of health care complexity, collaboration between professions is more important than ever before, and there is a strong move to new models of care that embed interprofessional practice as the norm (for a review, see Thistlethwaite, 2012). We argue that this move must not only focus 
on "training" health professionals in communication competency, but attention must be afforded to the differing cultures, rules, and professional norms of health professions, that make for a highly intergroup context. CAT is the ideal theoretical basis for interventions that shape and improve interprofessional practice. Next, we briefly describe how CAT can help us to understand how communication is influenced by the intergroup context and the communicative strategies that can be employed (see Chapter 7).

\section{Accommodative Positions and Strategies}

When individuals converse with each other, they often accommodate their behavior to ensure each understands the other and both view each other positively (see Chapter 3 ). By contrast, individuals may decide the encounter is not positive and take a nonaccommodative position. Such positions could include counteraccommodation, where a person is hostile and may constantly interrupt their speech partner. They may also choose to be patronizing (overaccommodation) and use a demeaning tone in their conversation (e.g., Ryan, Bajorek, Beaman, \& Anas, 2005; Ryan, Giles, Bartolucci, \& Henwood, 1986). These positions are often discussed alongside specific communication behaviors.

CAT describes five types of communication behaviors (also called strategies) that reflect the intergroup and/or interpersonal dynamics that occur in a given conversation (Gallois, Ogay, \& Giles, 2005). The first of these is approximation. This strategy describes the linguistic changes individuals make to their speech, and includes changes in accent, language, speech rate, and pauses. It concerns language production. For example, individuals may choose to 
converge linguistically toward their speech partner in order to gain that person's approval. In the health context, professionals may adapt their communication to meet the needs of the patient; for example, by talking more slowly or matching language use. The goal would be to ensure that the linguistic differences between the speakers are reduced. By contrast, a speaker may diverge from their speech partner and become more linguistically distant. This behavior may serve to exaggerate the differences between the two speakers. It may signal dislike by one speaker and, in many cases, serves to accentuate their different group memberships and demonstrate their different group identities.

A second strategy is interpretability, which relates to communicative competence and mutual understanding. The interpretability strategies used depends on the speaker's motivation and their perception of the other person. Judging a speech partner to be highly educated and proficient in medical knowledge may result in a health professional using medical terms in their conversation with a patient. If the assessment is accurate then the health professional will be engaging in appropriate interpretability and the patient's competence is matched. If not, such behavior may impair comprehension, impact on trust between patient and provider, and limit the likelihood of following recommendations for treatment.

A third strategy is discourse management. This strategy refers to the communication process rather than the content, and addresses the communication needs of the speakers. Behaviors such as back-channels, appropriate turn lengths, and sharing the way topics are chosen, introduced, and 
developed, ensure that each speaker is listened to and is engaged in the conversation.

The fourth strategy is interpersonal control, and focuses on the role relationship of each interactant. Interpersonal control is accomplished through the extent to which individuals remain in, or able to move outside of, their role. In the health context, health professionals may view the patient as a passive contributor to the conversation, and may use strategies such as interruption, abrupt topic change, and consultation termination to signal their control over the patient and reduce the patient's ability to express their opinions. High status health professionals may also employ these strategies when engaging with those who are junior to them. Alternatively, health professionals may engage in small talk to make an interaction more interpersonal, or seek a shared role or identity with a person, such as parent or sporting fan.

The final strategy is about emotional expression and focuses on the relational needs of the speakers. Appropriate emotional expression occurs when the other person's individual needs for reassurance are met and their concerns are addressed. Health professionals show appropriate emotional expression when they seek to reassure an anxious patient or are supportive of colleagues' needs.

Each of these five strategies can be used during interpersonal interactions, and may heighten or reduce intergroup differences. The value of CAT in explicating the intergroup and interpersonal relational dynamics in health care has a long history, and next we briefly review its application in health care communication research. 


\section{CAT Research in Health Professional and Patient Communication}

In the nineties and early 2000s, scholars started to investigate the intergroup and interpersonal dynamics that occur during health care consultations. A main focus was patients' perceptions of satisfactory and unsatisfactory interactions with health providers (Street, 1991, 2001; Watson \& Gallois, 1999, 2002). The findings confirmed that the intergroup dimension explained patients' perceptions of the interactions, and subsequent communication behavior and evaluations of their interactions. For example, the Watson and Gallois studies (1998, 1999, 2002) found that specific CAT communication strategies (interpersonal control, discourse management, and emotional expression) were strong predictors of patient satisfaction. Patients rated their interactions with health providers as most positive when they perceived that they had some control in the consultation, such as being able to check understanding and question the health professional's decision, when they actively engaged with the health professional, and when they felt reassured. Patients did not rate health providers negatively who were directive and exerted control over their patients, provided they also gave their patients opportunities to have control in the consultations. These findings demonstrated the interplay between interpersonal and intergroup dynamics in any given interaction. Interpretability was the one strategy that did not significantly predict patient satisfaction.

Farzadnia and Giles (2015) recently reviewed research that applied CAT to the health provider and patient domain, and demonstrated that CAT can be used as an intervention technique to change communication behavior. In eight 
studies examining patient's descriptions of pain to health professionals, they found that when health professionals engaged in CAT strategies, such as accommodative discourse management, patients were able to provide more specific information about their pain. Relatedly, in an investigation of end of life doctor-patient interactions, Ianssen and MacLeod (2010) reported that patients valued doctors who accommodated to their needs and focused on them as individuals with their own unique concerns.

By contrast, other studies demonstrated the negative effects of nonaccommodation with older adults or patients with a communication disability. When health professionals used patronizing speech, high levels of interpersonal control, and counteraccommodation, it reduced perceptions of quality of life for older adults in care (Lagacé, Tanguay, Lavallée, Laplante, \& Robichaud, 2012). Nurses at one care facility reported that they used nonaccommodative strategies because of time constraints, and admitted frustration with their ability to make themselves understood with older adults (Hemsley, Balandin, \& Worrall, 2012). Williams (2006) evaluated an intervention to reduce such communication behaviors, and showed that while improvement occurred after the intervention, with health providers being less controlling, more respectful, and more caring, health providers regressed to their previous communication patterns after two months.

CAT has a significant role in examining communication between patients and health professionals when they are from different cultures. In interviews with twelve physicians, Iain and Krieger (2011) highlighted the communication barriers experienced by doctors from a different culture, and described their 
strategies to overcome them. These included learning American slang, practicing American pronunciation, and changing speech rate. Scholl, Wilson, and Hughes (2011) found that physicians and patients downplayed their ethnic identities as factors influencing communication; instead, doctors focused on the need for patients to be competent communicators (by clearly stating their medical concerns), and patients focused on doctors being linguistically competent (and speaking the patient's language) irrespective of the doctor's ethnicity. Gasiorek, Van de Poel, and Blockmans (2015) also invoked CAT to examine how doctors managed consultations in a multilingual hospital, and demonstrated that they used code-switching and nonverbal gestures to make themselves better understood and to promote positive affiliation when language was a barrier. In sum, CAT has the ability to unpack a variety of communication strategies that interactants engage in to achieve communication competence.

Studies on nurse-parent communication show how CAT can be useful in explicating the differences between effective and ineffective communication, how members of different social groups value different strategies, and how we need to consider the interplay between multiple social group memberships. Iones, Woodhouse, and Rowe (2007) used CAT to explore parents' perceptions of effective and ineffective communication with nurses in a neonatal nursery and Dordic (2009) compared mothers' and nurses' perceptions. These studies found that effective communication was generally described as accommodative and ineffective communication was most frequently described as underaccommodative but, occasionally, described as overaccommodative. For effective communication, discourse management and emotional expression were 
the most frequently mentioned strategies, and to a lesser extent interpretability. Both studies showed ineffective communication is not just the opposite of effective communication, but is characterized by nonaccommodative use of strategies such as interpretability, discourse management, interpersonal control, and face (the public self-image of people). For example, comments included being spoken down to or treated as if they were silly, being scolded, and nurses who treated parents as irrelevant or just another parent. These studies also showed how different group memberships interact; for example, Iones et al. (2007) found that mothers mentioned negative face and interpersonal control more when describing ineffective communication, whereas fathers mentioned more the interpretability strategy.

Another interesting line of enquiry that further extends CAT in the health professional and patient domain is research undertaken to explore the role of concordance or discordance of explanatory models (EMs) between doctors and their patients who are being treated for depression. Doctors may believe that the cause of depression (EM) is a chemical imbalance or the result of psychosocial pressures (a different EM), and their belief may differ from that of their patient. This ongoing work investigates the role of effective communication, as operationalized through CAT, in establishing the patient and doctor alliance even when EMs are discordant (Teh, 2014).

Despite the research we have reviewed here on patient-practitioner interactions, there are still relatively few papers explicitly invoking CAT to design their research. Instead, many studies interpretively allude to concepts 
and strategies, such as approximation or adaptive behaviors, and the role of social identities.

\section{Communication Between Health Care Professionals}

There has also been a growing body of research examining communication between health professions from a CAT perspective. This expansion resulted from the mounting evidence that communication between health professionals was often ineffective, and was leading to patient harm (e.g., Leonard et al., 2004; Sutcliffe, Lewton, \& Rosenthal, 2004). The unique contribution of CAT research in this context is the explicit recognition of intergroup encounters between health professionals, that is encounters that are interpersonal (i.e., person-toperson) but that typically occur at an intergroup level (Dragojevic \& Giles, 2014; Hewett, Watson, Gallois, Ward, \& Leggett, 2009a, b).

For example, Setchell, Leach, Watson, and Hewett (2015) recently demonstrated strong resistance by doctors to allowing nurses to take on some of the work traditionally conducted by doctors. Importantly, the study showed that doctors used highly derogatory language about the outgroup (nurses) to justify their non-support of the proposal. One doctor wrote: “... What is happening to our standards to even consider this nonsense!!!????? Shouldn't we be training our own trainees first before wasting time on nurse endoscopy!!!!!!!!!!!!!!!!!!!!!!!” It appeared that doctors felt highly threatened by the proposed change, and responded (as CAT would predict) by denigrating the nurses (the outgroup). 
Much of the recent research has focused on hospital settings as a key context for investigating health communication. As we have noted earlier, hospitals are complex hierarchical environments, where a diverse range of health professionals work together. They are also organizations, where organizational identities drive behavior. Communication scholars have applied this intergroup framework to investigate how individuals negotiate and communicate their professional identities in organizations (see Gardner, Paulsen, Gallois, Callan, \& Monaghan, 2001). This framework is particularly relevant where there is tension and threat between groups. The numerous professional identities that co-exist in hospitals, and the tensions between them because of status and power differentials, combined with the continual cutting of resources (staffing and finance), makes this an environment where "tribal" warfare plays out between the professions (Bartunek, 2010; Hewett et al., 2009a, b; Lingard, Espin, Evans, \& Hawryluck, 2004; Lingard, Schryer, Spafford, \& Campbell, 2007).

The intergroup climate (termed "silo" mentality) prevents effective communication and information exchange. Patients can be caught in the "crossfire of intergroup dynamics" (Watson et al., 2012, p. 294). CAT is ideally positioned to unpack the intergroup dynamics of interactions between health professionals, because it explicitly explores the motivations and cognitions that make specific group identities salient, and shape our perceptions and behavior in a given interaction (see Chapter 3 ).

Hewett and colleagues found clear intergroup conflicts between doctors from different subspecialties ( Hewett, Watson, \& Gallois, 2015; Hewett et al., 
2009a, b). Importantly, they found that patient care was compromised because of the intergroup dynamics, which influenced communication between doctors from various specialties. Hospital structural factors precluding shared patient care, together with ambiguities in hospital processes and policy, were central to the generation of an intergroup climate. Hewett and colleagues showed how intergroup rivalry led to tribal allegiance and "turf" wars (intergroup conflict) between specialties, which negatively influenced, or took priority over, the negotiation of patient care. For example, posturing between departments resulted in threats to withhold patient care. In written communication, underaccommodation was ubiquitous. Through the interpretability strategy, doctors underaccommodated when communicating their findings and recommendations, so that specialty-specific ingroup language was incorrectly interpreted by doctors from outgroup specialties, leading to inappropriate recommendations for subsequent patient care. When conflict occurred, doctors used nonaccommodative strategies to secure intergroup dominance, by communicating, in writing, a shift in responsibility for patient care, and their dissatisfaction with other specialties.

Clinical handover/handoff (the transfer of patient information between hospital staff, usually when one shift ends and new staff take over, or when patients move from one ward to unit to another) has been identified as a weak link in patient care (Thomas, Schultz, Hannaford, \& Runciman, 2013). This is an ideal area for CAT to explore, as it provides insights into the barriers around handovers. Watson, Jones, and Cretchley (2014) asked different professions what were the communication strengths and problems for effective clinical 
handover. Two issues emerged. First, the different professions all discussed the same issues around handover, including time management, time pressures, and the difficulties of coordinating different handovers. Second, they found that each professional group had its own unique perceptions and priorities about handovers. The findings from this study suggested that health professionals understood what was required for handover improvement, but did not believe they had capacity to influence and change their working environment. Watson et al. (2014) concluded that handover scheduling by clinician managers needed to prioritize interprofessional representation.

Watson, Manias, Geddes, Della, and Iones (2015) investigated handover issues across a number of hospitals, and concluded that outside expertise from different research disciplines is needed to change the current practices and culture. They found a strong intergroup culture, where professional identities possessed their own rules and regulations, and the silo mentality was evident. In this environment, communication between professions was often ineffective and important patient information was not always conveyed. They suggested that clinicians work with communication and interdisciplinary scholars to bring about system improvement.

Watson et al.'s (2015) study invoked a model of miscommunication that outlines different types/levels of miscommunication (Coupland, Wiemann, \& Giles, 1991). Although not exhaustive, this model provides possible answers as to why improvements and changes in handover communication are not happening. For example, Coupland and colleagues suggested a lack of awareness by those working inside organizations to recognize the systems problems they 
face. To remedy this, Coupland and colleagues proposed that interdisciplinary scholars from outside an organization (e.g., outside the hospital system) should collaborate with key stakeholders (e.g., clinicians and management) to negotiate cultural change.

Intergroup dynamics in health care extend beyond simple ingroup and outgroup relations. Grice, Gallois, Jones, Paulsen, and Callan (2006) demonstrated the existence of double, "nested" or "cross-cutting" identities in a large public hospital. This refers to individuals who share the same work team and occupational profession (nurse and nurse on same team) as opposed to sharing the same work team, but belonging to a different professional group (e.g., nurse and physical therapist on same team). In their study of employees at a psychiatric hospital, they found that individuals within the same work team and same profession felt they provided the same detail of information to the double ingroup identity colleagues as well as to those who were in their team but belonged to a different profession. In contrast, they perceived that information relayed to them was more detailed when given by a double ingroup identity colleague than when delivered by someone who was in the same team but not the same profession. This finding highlights the intricacies of team and professional identification, and its influence on perceived health communication. More research is needed to explore how double and nested identities influence communication, particularly in complex social systems such as hospitals. What is clear is that this intergroup phenomenon lies outside of communication skills training and goes to the heart of how individuals are motivated to communicate from a sociopsychological perspective. 


\section{Emerging Methods}

More recently, we have also seen the application of a range of new methods of analysis to CAT research on health communication, providing us with different ways to unpack communication. Most of this research has focused on interactions between health professionals and patients. D'Agostino and Bylund (2013) used the Nonverbal Accommodation Coding System (NASS) to explore nonverbal approximation strategies between physicians and patients. They showed that, in over 50 per cent of cases, physicians did not converge to the patients' nonverbal behaviors. This new tool expands our understanding of approximation beyond verbal behavior, and has great potential for further unpacking the complexities of effective accommodation.

Angus, Watson, Gallois, and Wiles (2012) examined verbal communication in simulated doctor and patient interactions using a software package called Discursis (see Chapter 6). This software visualizes conversations across time by showing how interactants share topics and manage the conversation. Angus et al. found that when there was evidence of a lack of accommodation in at least one of the identified CAT strategies, patients were less satisfied with the consultation. Their examination of conversations highlighted how engagement and a balance of task and rapport were critical elements of good consultative communication. Baker and colleagues (2015) examined communication exchanges between carers and dementia patients. Visualization of the interactions with Discursis demonstrated how specific communication strategies influence patient engagement. When carers used reflection, it improved engagement on the part of the patient. Conversely, when carers did not 
listen attentively to patients, or did not allow the patient sufficient time to respond, there was reduced engagement.

Watson, Angus, Gore, and Farmer (2015) employed the same technology to examine training sessions between health professionals and simulated patients or family members. The health professional was required to explain to a patient or family member the reasons why they had experienced an adverse event. Watson et al.'s study demonstrated how the use of accommodative CAT strategies resulted in more effective open disclosure encounters with patients and families. In this difficult context, effective encounters were defined as both parties being able to move toward resolution. The Watson et al. study also linked approximation with sociopsychological strategies to demonstrate the effect approximation has on communication when employed by the health professional or by the family or patient.

A number of researchers have used the software tool Leximancer (Smith, 2003) to examine a range of health provider and patient interactions (e.g., Baker, Gallois, Drieger, \& Santesso, 2011; Baker \& Watson, 2015). Baker and Watson asked Canadian and Australian patients to reflect on their consultations with doctors and nurses. They found that patients' perceptions of whether their consultation was a negative or positive experience were directly related to the health providers' communication strategies. When health providers listened to the patients and checked understanding, patients felt more comfortable with the consultation. Conversely, patients reported feeling ignored or patronized when they perceived that the health provider did not spend time listening to them and was not being actively attentive. Thus, CAT communication strategies that 
reflected communication competence were predictors of patient willingness to communicate.

These new lines of analysis provide new techniques to view the word choices individual make as they describe their beliefs and attitudes. In the case of Leximancer, concept maps are created that can be objectively derived without the researcher's theoretical input. Similarly, Discursis provides visualizations of conversations that do not depend on a theoretical framework. While these technologies can work as stand-alone tools, they also provide the communication scholar with opportunities for data triangulation.

\section{The Future of Health Communication}

This chapter has laid out the diversity of communication issues and challenges in health care. They span areas that move us beyond health professional and patient interactions, where this chapter began. But, we must not lose sight of the fact that embedded in all communication challenges, the patient is the common link - it is the reason why we as health communication scholars investigate intercultural language barriers, health professional silos, and associated hospital systems. Miscommunication, whatever the cause, can lead to poor patient care. Improving patient care through improved communication is now more critical than ever. Medical technology advances, and better standards of living in the western world, mean people are living longer, often with complex comorbidities that require the involvement of multiple health providers. In turn, this has seen a move to models of care emphasizing interprofessional practice or 
multidisciplinary teams. Often, however, these teams do not communicate competently, and the patient suffers.

It is disheartening in twenty-first century health communication scholarship that communication failure remains the most cited reason for poor patient outcomes. Recent health sector improvement efforts have focused on improving communication competency through skills training, and introducing communication tools that provide professionals with structured checklists. It is appears that, despite these interventions, there has been no reduction in adverse patient events. We have argued that a dogged focus on a theoretical skills training alone will not change the dynamics around health communication. Pitts and Harwood (2015) proposed that exploring communication accommodation competence is a clear direction for CAT researchers, which is one that resonates with us as health communication scholars.

The challenge for CAT scholars is there is no set template for communication effectiveness. It depends upon the type of interaction, the needs of the interactants, and the values placed on different strategies by different groups. This fact has always been embedded in CAT. Gasiorek (2015) proposed that health communication research must take account of both interactant identity/ies (and whether they are motivated to accentuate group distinctiveness in their communication) and context, which in health includes an array of factors such as the hospital culture, resource and time constraints, and other systemic factors. Pitts and Harwood (2015) set us the challenge of now actively revisiting CAT to use it for a task to which it is eminently suited, a theory 
of communication accommodation competence, where individuals establish competent relations.

We believe the time is right to showcase CAT as a framework to achieve communication competence. All the studies discussed in this chapter sought to explain the motivations and cognitions that underpinned communication problems. Some of the papers also examined the contexts around the interactions, and how issues such as time constraints, poor resources, intergroup conflicts, and the silos that exist for hospital clinicians and management, influence communication behaviors. Some of the papers reviewed here were intervention studies (e.g., Williams, 2006). It is good to see that some intervention work has begun. However, such studies need to look at the identities and the context as reasons for communication failure. CAT can unpack each of these and explore the issues around the miscommunication. The message from Pitts and Harwood (2015) gives us direction for advancing CAT. They say we must look at how individuals developing accommodation competence is a lifetime journey, and the health context provides a specific area for this research. Development of a communication accommodation based model of communication competence in health care would shift the focus of CAT toward application and intervention that may improve health care and patient outcomes.

We propose the following areas for future study that together will assist in developing a model of CAT competence in health care. First, such a model must incorporate the advances in health care. With the evolution of health care teams, we need to extend our attention beyond dyads to the communication strategies within interprofessional teams, with the associated power 
differentials. The role of the patient as part of that team is a critical part of the extended CAT model. Issues to be explored here are how leadership and decision making play out across the different health contexts. We need too a better understanding of the intergroup and interpersonal balance in such communication.

Second, E-health and remote care centers are becoming more common and have their own communication challenges that need our attention. To date, CAT has not examined mediated health communication and this area requires CAT application. The written medical patient record, whether paper or electronic, is also a fruitful line of enquiry which, to date, has had little work conducted on.

Third, CAT has a prominent role to play in the education of health professionals to achieve communication accommodation competence. The potential for better understanding patient care outcomes is integrally linked with the communication patterns of the teams making patient care decisions. Interprofessional education and practice are features of health care in the twenty-first century. Exploring team relations, and how they operate under different circumstances, such as different types of stress the team experience (patient deterioration, time constraints, and resource constraints), are key communication directions. Relatedly, we must use CAT to investigate the patient's journey, which consists of multiple conversations with a number of different healthcare professionals. This would provide us with insights into how a patient's accommodative stance or choice of strategies (by both themselves and by health professionals) change over time and as they interact with different 
health professionals. Finally, it is time to test interventions that invoke CAT, to examine how such interventions change communication behaviors, and improve interprofessional practice and patient quality of care.

The number of papers in health communication that incorporate CAT is a cause for celebration. CAT researchers have moved from focusing on health professional and patient interactions to examining interprofessional communication. Much of the research identified the interpersonal and intergroup dimensions of healthcare communication, and how communication accommodation strategies explain the effectiveness or otherwise of interactions about health, and the outcomes for patients, such as satisfaction. CAT has progressed our understanding of particular strategies that health professionals should be using to achieve competent communication. Accommodative use of approximation, discourse management, interpersonal control, and emotional expression by health professionals are associated with positive patient relations and willingness to engage (Baker \& Watson, in press). This body of research provides valuable insights for future research directions.

The health domain is expanding. New models of interprofessional care and medical advances are changing patient care. However, the perennial problems of communication failure and associated adverse events are not reducing. CAT has shown its value in exploring and explaining communication failure. The next step is to extend CAT as a vehicle for reducing communication failure in the health context. CAT has the potential to introduce a new pedagogy to communication accommodation competence training for health professionals, 
allowing new understanding for health professionals and patients about the complex system within which they communicate.

\section{chapter-references}

\section{References}

Angus, D., Watson, B. M., Gallois, C., \& Wiles, J. (2012). Visualizing conversation structure across time: Insights into effective doctor-patient consultations. PLoS One, 7, e38014.

Baker, R., Angus, D., Smith-Conway, E. R., Baker, K. S., Gallois, C., et al. (2015). Visualizing conversations between care home staff and residents with dementia. Ageing and Society, 32, 270-297.

Baker, S., Gallois, C., Drieger, S. M., \& Santesso, N. (2011). Communication accommodation and managing musculoskeletal disorders: Doctors' and patients' perspectives. Health Communication, 26, 379-388.

Baker, S. C., \& Watson, B. M. (2015). How patients perceive their doctors' communication: Implications for patient willingness to communicate. Journal of Language and Social Psychology, 34, 621-639.

Bartunek, J. M. (2010). Intergroup relationships and quality improvement in healthcare. British Medical Journal: Quality and Safety, 20, i62-i66.

Cargile, A. C., \& Giles, H. (1996). Intercultural communication training: Review, critique, and a new theoretical framework. In B. Burleson (Ed.), Communication yearbook 19 (pp. 385-423). Thousand Oaks, CA: Sage. 
Coiera, E. (2011). Why system inertia makes health reform so difficult. British Medical Journal, 342, 27-29.

Coupland, N., Wiemann, J. M., \& Giles, H. (1991). Talk as "problem" and communication as "miscommunication." An integrative analysis. In N. Coupland, H. Giles, \& J. M. Wiemann (Eds.), “Miscommunication" and problematic talk (pp. 1-17). Newbury Park, CA: Sage.

D’Agostino, T. A., \& Bylund, C. L. (2013). Nonverbal accommodation in health care communication. Health Communication, 29, 563-73.

Dordic, T. (2009). Mothers' and nurses' perceptions of communication in a neonatal special care unit: A communication accommodation theory approach. PhD, Brisbane, Australia: Griffith University.

Dragojevic, M., \& Giles, H. (2014). Language and interpersonal communication: Their intergroup dynamics. In C. R. Berger (Ed.), Handbook of interpersonal communication (pp. 29-51). Berlin, Germany: De Gruyter Mouton.

Emry, R., \& Wiseman, R. L. (1987). An intercultural understanding of ablebodied and disabled persons' communication. International Journal of Intercultural Relations, 11, 7-27.

Farzadnia, S., \& Giles, H. (2015). Patient-provider interaction: A communication accommodation theory perspective. International Journal of Society, Culture and Language, 3, 17-34. 
Gallois, C., Ogay, T., \& Giles, H. (2005). Communication accommodation theory: A look back and a look ahead. In W. Gudykunst (Ed.), Theorizing about intercultural communication (pp. 121-148). Thousand Oaks, CA: Sage.

Gardner, M. J., Paulsen, N., Gallois, C., Callan, V. J., \& Monaghan, P. (2001). Communication in organizations. An intergroup perspective. In W. P. Robinson, \& H. Giles (Eds.), The new handbook of language and social psychology (pp. 561-584). Chichester, UK: Wiley.

Gasiorek, J. (2015). Epilogue: Engaging identity and foregrounding context in health communication research. Journal of Language and Social Psychology, 34, 702-708

Gasiorek, J., Van de Poel, K., \& Blockmans, I. (2015). What do you do when you can't accommodate? Managing and evaluating problematic interactions in a multilingual medical environment. Language and Communication, 41, 84-88.

Grice, T. A., Gallois, C., Jones, E., Paulsen, N., \& Callan, V. J. (2006). “We do it, but they don't": Multiple categorizations and work team communication. Journal of Applied Communication Research, 34, 331-348.

Hemsley, B., Balandin, S., \& Worrall, L. (2012). Nursing the patient with complex communication needs: Time as a barrier and a facilitator to successful communication in hospital. Journal of Advanced Nursing, 68, 116-126.

Hewett, D. G., Watson, B. M., \& Gallois, C. (2015). Communication between hospital doctors: Underaccommodation and interpretability. Language and Communication, 41, 71-83. 
Hewett, D. G., Watson, B. M., Gallois, C., Ward, M., \& Leggett, B. A. (2009a).

Communication in medical records: Intergroup language and patient care. Journal of Language and Social Psychology, 28, 119-138.

Hewett, D. G., Watson, B. M., Gallois, C., Ward, M., \& Leggett, B. A. (2009b). Intergroup communication between hospital doctors: Implications for quality of patient care. Social Science and Medicine, 69, 1732-1740.

Jain, P., \& Krieger, J. L. (2011). Moving beyond the language barrier: The communication strategies used by international medical graduates in intercultural medical encounters. Patient Education and Counseling, 84, 98-104.

Janssen, A. L., \& MacLeod, R. D. (2010). What can people approaching death teach us about how to care? Patient Education and Counseling, 81, 251-256.

Jones, L., Woodhouse, D., \& Rowe, J. (2007). Effective nurse parent communication: A study of parents' perceptions in the nicu environment. Patient Education and Counseling, 69, 6.

Kreindler, S. A., Dowd, D. A., Dana Star, N., \& Gottschalk, T. (2012). Silos and social identity: The social identity approach as a framework for understanding and overcoming divisions in health care. Milbank Quarterly, 90, 347-374.

Lagacé, M., Tanguay, A., Lavallée, M. L., Laplante, J., \& Robichaud, S. (2012). The silent impact of ageist communication in long term care facilities: Elders' perspectives on quality of life and coping strategies. Journal of Aging Studies, 26, 335-342. 
Leonard, M., Graham, S., \& Bonacum, D. (2004). The human factor: The critical importance of effective teamwork and communication in providing safe care. Quality and Safety in Health Care, 13, i85-i90.

Lingard, L., Espin, S., Evans, C., \& Hawryluck, L. (2004). The rules of the game: Interprofessional collaboration on the intensive care unit team. Critical Care, 8, 403-408.

Lingard, L., Schryer, C. F., Spafford, M. M., \& Campbell, S. L. (2007). Negotiating the politics of identity in an interdisciplinary research team. Qualitative Research, 7, 501-519.

Ong, M. S., \& Coiera, E. (2011). A systematic review of failures in handoff communication during intrahospital transfers. Joint Commission Journal on Quality and Patient Safety, 37, 274-284.

Peters, K., Morton, T., \& Haslam, A. (2010). Communication silos and social identity complexity in organisations. In H. Giles, S. Reid, \& J. Harwood (Eds.), The dynamics of intergroup communication (pp. 221-233). New York: Peter Lang.

Pitts, M. J., \& Harwood, J. (2015). Communication accommodation competence: The nature and nurture of accommodative resources across the lifespan. Language and Communication, 41, 89-99.

Ryan, E. B., Bajorek, S., Beaman, A., \& Anas, A. P. (2005). “I just want you to know that 'them' is me": Intergroup perspectives on communication and disability. In J. Harwood, \& H. Giles (Eds.), Intergroup communication: Multiple perspectives (pp. 117-140). New York, NY: Peter Lang. 
Ryan, E. B., Giles, H., Bartolucci, G., \& Henwood, K. (1986). Psycholinguistic and social psychological components of communication by and with the elderly. Language and Communication, 6, 1-24.

Scholl, J. C., Wilson, J. B., \& Hughes, P. C. (2011). Expression of patients' and providers' identities during the medical interview. Qualitative Health Research, 21, 1022-1032.

Setchell, J., Leach, L. E., Watson, B. M., \& Hewett, D. G. (2015). Impact of identity on support for new roles in health care: A language inquiry of doctors' commentary. Journal of Language and Social Psychology, 34, 672-686.

Smith, A. E. (2003, May-June). Automatic extraction of semantic networks from text using leximancer. Paper presented at the HLT-NAACL 2003 Human Language Technology Conference of the North American Chapter of the Association for Computational Linguistics, Edmonton, Alberta, Canada.

Street, R. L., Jr. (1991). Accommodation in medical consultations. In H. Giles, J. Coupland, \& N. Coupland (Eds.), Contexts of accommodation: Developments in applied sociolinguistics (pp. 131-156). New York, NY: Cambridge University Press.

Street, R. L., Jr. (2001). Active patients as powerful communicators. In W. P. Robinson \& H. Giles (Eds.), The new handbook of language and social psychology (pp. 541-561). Chichester, UK: Wiley.

Street, R. L., Jr., \& Wiemann, J. M. (1988). Differences in how physicians and patients perceive physicians' relational communication. The Southern Speech Communication Journal, 53, 420-440. 
Sutcliffe, K. M. P., Lewton, E. P. M. P. H., \& Rosenthal, M. M. P. (2004).

Communication failures: An insidious contributor to medical mishaps. Academic Medicine Special Theme: Physician -Patient Relationships, 79, 186-194.

Teh, J. L. (2014, June). How do patients and doctors use depression explanatory models (EMs)? The role of EMs in communication effectiveness, selfstigma levels and treatment satisfaction. Paper presented at the ICLASP14, Honolulu, USA.

Thistlethwaite, J. (2012). Interprofessional education: A review of context, learning and the research agenda. Medical Education, 46, 58-70.

Thomas, M. J. W., Schultz, T. J., Hannaford, N., \& Runciman, W. B. (2013). Failures in transition: Learning from incidents relating to clinical handover in acute care. Journal For Healthcare Quality, 35, 49-56.

Vogus, T. J., Sutcliffe, K. M., \& Weick, K. E. (2010). Doing no harm: Enabling, enacting, and elaborating a culture of safety in health care. Academy of Management Perspectives, 24, 60-77.

Watson, B. M., Angus, D., Gore, L., \& Farmer, J. (2015). Communication in open disclosure conversations about adverse events in hospitals. Language and Communication, 41, 57-70.

Watson, B. M., \& Gallois, C. (1998). Nurturing communication by health professionals toward patients: A communication accommodation theory approach. Health Communication, 10, 343-355. 
Watson, B. M., \& Gallois, C. (1999). Communication accommodation between patients and health professionals: Themes and strategies in satisfying and unsatisfying encounters. International Journal of Applied Linguistics, 9, 167-184.

Watson, B. M., \& Gallois, C. (2002). Patients' interactions with health providers: A linguistic category model approach. Journal of Language and Social Psychology, 21, 32-52.

Watson, B. M., \& Gallois, C. (2007). Language, discourse, and communication about health and illness: Intergroup relations, role, and emotional support. In A. Weatherall, B. M. Watson, \& C. Gallois (Eds.), Language and discourse and social psychology (pp. 108-130). Basingstoke, UK: Palgrave Macmillan.

Watson, B. M., Gallois, C., Hewett, D. G., \& Jones, E. (2012). Culture and health care: Intergroup communication and its consequences. In J. Jackson (Ed.), The routledge handbook of intercultural communication (pp. 512-524). London, UK: Routledge.

Watson, B. M., Hewett, D. G., \& Gallois, C. (2012). Intergroup communication and healthcare. In H. Giles (Ed.), The handbook of intergroup communication (pp. 293-305). New York, NY: Routledge.

Watson, B. M., Jones, L., \& Cretchley, J. (2014). Time as a key topic in health professionals' perceptions of clinical handovers. Global Qualitative Nursing Research, 1, 1-11. 
Watson, B. M., Manias, E., Geddes, F., Della, P., \& Jones, D. (2015). An analysis of clinical handover miscommunication using a language and social psychology approach. Journal of Language and Social Psychology, 34, 687701.

Williams, K. N. (2006). Improving outcomes of nursing home interactions. Research in Nursing and Health, 29, 121-133.

Wright, K. B., Sparks, L., \& O’Hair, H. D. (2008). Health communication in the 21st century. Maldon: MA: Blackwell. 